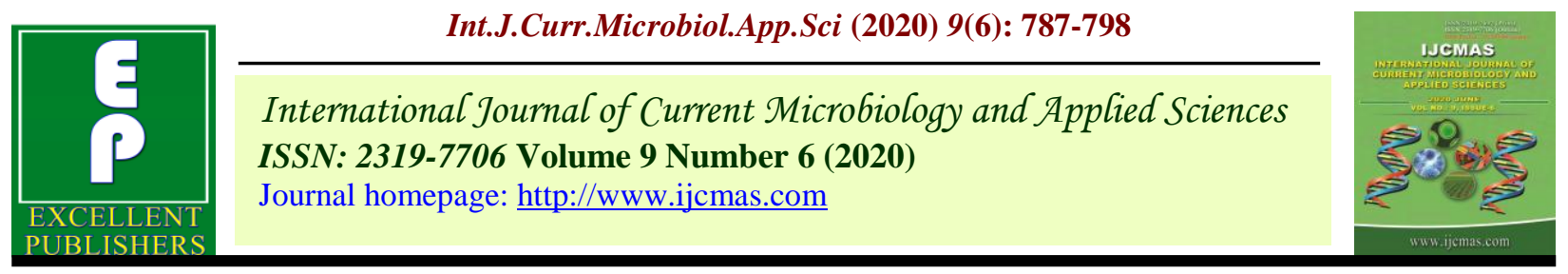

Case Study

https://doi.org/10.20546/ijcmas.2020.906.101

\title{
Production Potential Appraisal: A Case Study in Banaganapalle Mandal of Kurnool District in Andhra Pradesh, India
}

\author{
P.V. Geetha Sireesha* and M.V.S. Naidu \\ Department of Soil Science and Agricultural Chemistry, S.V. Agricultural College, Acharya \\ N.G. Ranga Agricultural University, Tirupati, Andhra Pradesh, India \\ *Corresponding author
}

\begin{tabular}{l} 
Ke y w o r d s \\
Soil productivity, \\
Potential \\
productivity, \\
Coefficient of \\
improvement \\
\hline Article Info \\
\hline $\begin{array}{l}\text { Accepted: } \\
\text { 16 May } 2020 \\
\text { Available Online: } \\
\text { 10 June } 2020\end{array}$ \\
\hline
\end{tabular}

\section{A B S T R A C T}

Plains (nearly level) and upland (very gentle sloping) topography, affected by erosion and moisture stress, is the unique feature of Banaganapalle region. Soils developed from dolomite and granite-gneiss parent material under varying land use were studied for their morphological characteristics, physical and physicochemical properties. These soils were slightly alkaline to strongly alkaline in reaction, non-saline, deep to very deep in depth. Soils were low to medium in available nitrogen, medium to high in available phosphorus, low to high in available potassium and high in available sulphur. The DTPA extractable $\mathrm{Zn}$ and Fe were deficient whereas DTPA extractable $\mathrm{Cu}$ and $\mathrm{Mn}$ were sufficient. All the soils of the study area fall under agricultural land with land capability classes ranging from II to IV. Further, the lands have limitations of slope and erosion. Soil productivity and productivity potentiality increased from plains to upland topography. Soil productivity class ranged from extremely poor or nil (13.38) to good (45.90) while the potential productivity of soil class ranged from Average (29.16) to excellent (90.00). The coefficient of improvement (Ci) varied from 1.85 to 3.92 indicating the implementation of judicious soil and water management practices suggested for their sustainable management of Banaganapalle mandal.

\section{Introduction}

Rainfed agriculture contributes $58 \%$ of the global food basket and constitutes $66 \%$ of the net sown area in India (Wani et al., 2009). In view of the present global crises on food, rising food prices in the international market, progressive conversion of good lands to grow bio-fuel crops and / or other non - agricultural uses, SEZ challenge, demand for urbanization and industrialization etc., technologies for efficient management of our finite soil resources in Agro-ecological regions is of utmost importance towards ensuring productivity, profitability and national food security (Sarkar, 2011). The risk of increasing degradation is expected to intensify in future due to increase in the human and animal 
population, ignorance of improved technologies. The predicted climate change may adversely affect the quality and productivity of the soil resource in the monsoon dependent areas of the state. To achieve soil resource management in Agroecological regions needs knowledge on morphological, physical and chemical characteristics and classification is an essential requirement. Banaganpalle mandal falls under semi-arid agro-ecological region and had a wide variety of soil resources. However, so far no efforts being made to study the genesis, classification and evaluation of soils, which had pivotal role not only to sustain soil fertility but also improved soil productivity. Hence, present study was taken up.

\section{Materials and Methods}

Banaganapalle mandal lies in between $15^{\circ} 08^{\prime}$ and $15^{\circ} 24^{\prime}$ North latitudes and $79^{\circ} 12^{\prime}$ and $78^{\circ} 16^{\prime}$ East longitudes. The study area consists of dolomite and granite-gneiss parent material (Table 1). The area of Banaganapalle mandal representing transition between young alluvial plains of this region. The climate belongs to semi-arid monsoonic with distinct summer, winter and rainy seasons. The mean annual rainfall recorded for the last 10 (20022011) years is $775 \mathrm{~mm}$ of which 90 per cent was received during June to September. The mean annual temperature was $28.7^{\circ} \mathrm{C}$ with mean summer temperature $33.5^{\circ} \mathrm{C}$ and the mean winter temperature of $28.7^{\circ} \mathrm{C}$. The maximum temperature recorded for last 10 years is $39^{\circ} \mathrm{C}$ and the minimum temperature $25^{\circ} \mathrm{C}$ in the month of December (Sehgal et $a l .$, 1992). The soil moisture regime has been computed as ustic and soil temperature regime as iso-hyperthermic. The natural vegetation comprises of Tridax procumbens, Parthenium hysterophorus, Prosopis juliflora, Calotropis gigantia, Acacia auriculiformis, Commalina bengalensis, Cynodon dactylon,
Cyprus rotundus, Pongamia pinneta, Azadirachta indica etc.

A reconnaissance soil survey was conducted in Banaganapalle mandal located in semiarid agro-ecological region using toposheet of 1:50,000 scale as per the procedure outlined by AIS \& LUS (1970). Auger bores, mini pits, road cuts and 12 pedons located on plains and uplands were studied. Soil correlation exercises yielded seven typical pedons i.e., four pedons in plains and three in uplands were identified for the study. These seven pedons were studied in detail and the morphological characteristics were presented in table 2. The detailed morphological descriptions of these seven pedons were studied in the field as per the procedure outlined in Soil Survey Manual (Soil Survey Division Staff 2000). Later, horizon-wise samples were collected and characterized for important physical, physico-chemical properties and available nutrient status using standard procedures. The soils were classified taxonomically (Soil Survey Staff 2010). Considering limitations and potentials of the soils, land capability classification was evaluated up to sub-class level (Klingebiel and Montgomery 1966) and based on that a suitable land use plan has also been suggested. Actual Productivity (P) was calculated with the parametric model developed by Riquier et al., (1970). In the model (Eq. 1) nine parameters were employed for assessment of soil productivity

$\mathrm{P}=\mathrm{H}$ X D X P X T X N/S X O X A X M ... (1)

where, $\mathrm{P}$ - production potential, H - soil moisture content, D - drainage, $\mathrm{P}$-effective soil depth, $\mathrm{T}$ - texture/structure, $\mathrm{N}$ - base saturation, S - soluble salts, O - organic matter, $\mathrm{A}$ - mineral exchange capacity and $\mathrm{M}$ - mineral reserves. After employing all the possible improvement factors, the potential 
productivity rating was worked out by assigning different grades. The coefficient of improvement $(\mathrm{CI})$ was worked out based on the actual productivity and potential productivity ratings as given below:

Coefficient of Improvement $(\mathrm{CI})=$

Potential productivity rating

Actual productivity rating

\section{Results and Discussion}

\section{Soil characteristics}

\section{Physical characteristics}

The detailed physical characteristics of the soils were presented in table 2. Granulometric data revealed that the clay content varied from 17.53 to 51.42 per cent. The increase in clay content in Bw horizon of pedons 1, 3, 4 and 5 were primarily due to in situ weathering of parent material, known through study of morphological properties. More or less decrease of clay content with depth was noticed in pedons 1,2 and 5, this might be due to variability of weathering in different horizons. Silt content in general exhibited an irregular trend with depth, this irregular distribution of silt might be due to variation in weathering of parent material or in situ formation (Satish Kumar and Naidu, 2012). Sand constitutes the bulk of mechanical fractions, which could be attributed to the siliceous nature of granite-gneiss parent material. The bulk density of different pedons varied from 1.15 to $1.78 \mathrm{Mg} \mathrm{m}^{-3}$ and had shown more or less an increasing trend with depth due to low organic matter and less aggregation. Similar findings were reported by Singh and Agarwal (2005). Low bulk density values of surface soils are due to higher organic matter content. This is evident from the significant negative correlation $(\mathrm{r}=$ 0.404) of bulk density with organic carbon at $5 \%$ level. Water holding capacity of different pedons varied from 27 to 70 per cent. These variations were due to the difference in depth, clay, silt and organic carbon content and low water holding capacity in sandy soils was due to high sand and less clay content as evident by negative correlation $(\mathrm{r}=-0.513)$ at $5 \%$ level between water holding capacity and sand content. The irregular trend of water holding capacity with depth was due to the illuviation and eluviation of finer fractions in different horizons.

\section{Physical and chemical characteristics}

All the pedons were slightly alkaline to strongly alkaline in reaction; this wide variation was attributed to the nature of the parent material, leaching, presence of calcium carbonate and exchangeable sodium. The difference between the $\mathrm{pH}_{\mathrm{KCl}}$ and $\mathrm{pH}_{\mathrm{H}_{2} \mathrm{O}}$ values $\left(\Delta \mathrm{pH}=\mathrm{pH}_{\mathrm{KCl}} \mathrm{pH}_{\mathrm{H}_{2} \mathrm{O}}\right)$ with large negative value (more than -0.5) indicated a high negative surface charge density in these soils. All the pedons had shown low to medium electrical conductivity values ranging from 0.12 to $0.93 \mathrm{dS} \mathrm{m}$, indicating non saline nature. The low electrical conductivity may be due to free drainage conditions which favoured the removal of released bases by percolating and drainage water (Table 2). Organic carbon content of these soils was found to be low to medium and ranging from 0.04 to 0.75 per cent (Table 2). The organic carbon content decreased with depth in almost all the pedons. This is attributed to the addition of plant residues and farmyard manure to surface horizons than in the lower horizons. The low organic matter content in the soils might be attributed to the prevalence of tropical condition, where the degradation of organic matter occurs at a faster rate coupled with low vegetation cover, there by leaving less organic carbon in the soils (Nayak et al., 2002).

The CEC in all the pedons estimated by ammonium acetate extract varied from 21.8 to 
$51.2 \mathrm{cmol}\left(\mathrm{p}^{+}\right) \mathrm{kg}^{-1}$ soil which corresponds to clay content in the horizons, organic carbon content and also type of clay mineral present in these soils. The free $\mathrm{CaCO}_{3}$ was ranged from 2.5 to 37.5 per cent and the highest $\mathrm{CaCO}_{3}$ content was noticed in pedons 3 and 5 which might be due to semi-arid climate which is responsible for the pedogenic processes resulting in the depletion of $\mathrm{Ca}^{+2}$ ions from the soil solution in the form of calcretes (Ashokkumar and Jagdish Prasad, 2010). However, in pedons 4, 6 and 7, the $\mathrm{CaCO}_{3}$ increased with depth which might be due to downward movement of calcium and its subsequent precipitation as carbonate and / or decomposition of calcium carbonate. Pedons 2, 4 and 7 showed an irregular distribution with depth, this may either be due to variable nature of geological material that contributed to these soils are to be rapid leaching of carbonates from the porous sandy soils (Singh and Agarwal 2005). Exchangeable bases in all pedons were in the order of $\mathrm{Ca}^{2+}>\mathrm{Mg}^{2+}>\mathrm{Na}^{+}>\mathrm{K}^{+}$on the exchange complex. The base saturation varied from 51 to 99 per cent. The higher exchangeable $\mathrm{Ca}$ in the surface soil may be due to redistribution of calcium by tree species (Patil and Jagdish Prasad 2004). The ratio between $\mathrm{Ca}$ and $\mathrm{Mg}$ ranged from 13.29 to 1.63 and narrower $\mathrm{Ca}^{+2} / \mathrm{Mg}^{+2}$ ratio was due to suppression of $\mathrm{Ca}$ solubility, substitution of $\mathrm{Mg}^{+2}$ or $\mathrm{Ca}^{+2}$ by plants and recycling of unusual amount of $\mathrm{Mg}$. similar results were reported by (Raghuwanshi et al., 2011). The ratio between CEC and clay ranged from 1.87 to 0.82 and the CEC: clay ratio was used to identify the clay mineralogy (Ashokkumar and Jagdish Prasad, 2010).

\section{Nutrient status and soil fertility}

\section{Macronutrients}

Soil fertility exhibits the status of different soils with regard to the amount and availability of nutrients essential for plant growth. The available nitrogen content varied from 190 to $439 \mathrm{~kg} \mathrm{ha}^{-1}$ (Table 4) throughout the depth. However, available nitrogen content found to be maximum in surface horizons and decreased regularly with depth which is due to decreasing trend of organic carbon with depth and cultivation of crops are mainly confined to the surface horizon (Rhizosphere) only and at regular interval the depleted nitrogen content is supplemented by the external addition of fertilizers during crop cultivation (Satish Kumar and Naidu, 2012).

The available phosphorus varied from 13 to $78 \mathrm{~kg} \mathrm{ha}^{-1}$ in different revenue villages in this mandal. However, the highest available phosphorus content was observed in the surface horizons and decreased with depth. It might be due to the confinement of crop cultivation to the rhizosphere and supplementing the depleted phosphorus by external sources i.e., fertilizers and presence of free iron oxide and exchangeable $\mathrm{Al}^{3+}$ in smaller amounts (Thangasamy et al., 2005). The lower phosphorus content in sub-surface horizons compared to surface horizon was due to the fixation of released phosphorus by clay minerals and oxides of iron and aluminum. Available potassium content of soils varies from 44 to $409 \mathrm{~kg} \mathrm{ha}^{-1}$.

The highest available potassium content was observed in the surface horizons and showed more or less decreasing trend with depth. This might be attributed to more intense weathering, release of liable $\mathrm{K}$ from organic residues, application of $\mathrm{K}$ fertilizers and upward translocation of potassium from lower depths along with capillary raise of ground water (Sharma and Anil Kumar, 2003). The available sulphur in soils varied from 13 to 68 $\mathrm{mg} \mathrm{kg} \mathrm{kg}^{-1}$, due to higher amount of organic matter in surface layers than in deeper layers the available sulphur is more in surface horizons than sub-surface horizons. 
Table.1 Landscape characteristics of pedons

\begin{tabular}{|c|c|c|c|c|c|c|}
\hline Pedons / Villages & Location & $\begin{array}{l}\text { Elevation } \\
\text { above MSL } \\
\text { (m) }\end{array}$ & Mineralogy & $\begin{array}{l}\text { Slope } \\
(\%)\end{array}$ & Drainage & $\begin{array}{c}\text { Parent } \\
\text { material }\end{array}$ \\
\hline P1 Pasupula & $\begin{array}{l}15^{\circ} 08^{\prime} 04.5^{\prime \prime} \mathrm{N} \\
78^{\circ} 16^{\prime} 48.3^{\prime \prime} \mathrm{E}\end{array}$ & 243 & $\begin{array}{l}\text { Fine-loamy, smectitic, } \\
\text { iso-hyperthermic, Vertic } \\
\text { Haplustepts }\end{array}$ & $0-1$ & $\begin{array}{c}\text { Somewhat poorly } \\
\text { drained }\end{array}$ & Granite-gneiss \\
\hline P2 Yerragudi Palem & $\begin{array}{l}15^{\circ} 23^{\prime} 00.9^{\prime \prime} \mathrm{N} \\
78^{\circ} 12^{\prime} 26.3^{\prime \prime} \mathrm{E}\end{array}$ & 274 & $\begin{array}{l}\text { Fine-loamy, smectitic, } \\
\text { iso-hyperthermic, Typic } \\
\text { Ustorthents }\end{array}$ & $0-1$ & $\begin{array}{c}\text { Moderately well } \\
\text { drained }\end{array}$ & Granite-gneiss \\
\hline P3 Palukuru & $\begin{array}{l}15^{\circ} 24^{\prime} 00.1^{\prime \prime} \mathrm{N} \\
78^{\circ} 12^{\prime} 03.9^{\prime \prime} \mathrm{E}\end{array}$ & 287 & $\begin{array}{l}\text { Fine, smectitic, iso- } \\
\text { hyperthermic, Vertic } \\
\text { Haplustepts }\end{array}$ & $0-1$ & Poor & Dolomite \\
\hline P4 Mittepalle & $\begin{array}{l}15^{\circ} 18^{\prime} 57.4^{\prime \prime} \mathrm{N} \\
78^{\circ} 14^{\prime} 37.1^{\prime \prime} \mathrm{E}\end{array}$ & 233 & $\begin{array}{l}\text { Fine-loamy, smectitic, } \\
\text { iso-hyperthermic, Typic } \\
\text { Haplustepts }\end{array}$ & $1-3$ & $\begin{array}{c}\text { Somewhat poorly } \\
\text { drained }\end{array}$ & Granite-gneiss \\
\hline P5 Kapulapalle & $\begin{array}{l}15^{\circ} 18^{\prime} 12.6^{\prime \prime} \mathrm{N} \\
78^{\circ} 14^{\prime} 56.4^{\prime \prime} \mathrm{E}\end{array}$ & 230 & $\begin{array}{l}\text { Fine-loamy, kaolinitic, } \\
\text { iso-hyperthermic, } \\
\text { Fluventic Haplustepts }\end{array}$ & $0-1$ & $\begin{array}{c}\text { Somewhat poorly } \\
\text { drained }\end{array}$ & Granite-gneiss \\
\hline P6 Illuru Kothapeta & $\begin{array}{l}15^{\circ} 17^{\prime} 10.0^{\prime \prime} \mathrm{N} \\
78^{\circ} 15^{\prime} 31.9^{\prime \prime} \mathrm{E}\end{array}$ & 223 & $\begin{array}{l}\text { Fine, smectitic, iso- } \\
\text { hyperthermic, Lithic } \\
\text { Ustorthents }\end{array}$ & $1-3$ & $\begin{array}{c}\text { Somewhat poorly } \\
\text { drained }\end{array}$ & Granite-gneiss \\
\hline P7 Gulamnabipeta & $\begin{array}{l}15^{\circ} 15^{\prime} 44.3^{\prime \prime} \mathrm{N} \\
78^{\circ} 15^{\prime} 37.9^{\prime \prime} \mathrm{E}\end{array}$ & 216 & $\begin{array}{l}\text { Fine-loamy, kaolinitic, } \\
\text { iso-hyperthermic, Typic } \\
\text { Ustifluvents }\end{array}$ & $1-3$ & $\begin{array}{c}\text { Well } \\
\text { drained }\end{array}$ & Granite-gneiss \\
\hline
\end{tabular}


Table.2 Physico-chemical properties of the soils

\begin{tabular}{|c|c|c|c|c|c|c|c|c|c|c|c|c|}
\hline \multirow[t]{2}{*}{$\begin{array}{l}\text { Pedon No. } \\
\text { \& Horizon }\end{array}$} & \multirow[t]{2}{*}{$\begin{array}{l}\text { Depth } \\
\text { (m) }\end{array}$} & \multicolumn{2}{|c|}{ pH $(1: 2.5)$} & \multirow[t]{2}{*}{$\begin{array}{l}\text { EC } \\
\left(\text { dsm }^{-1}\right)\end{array}$} & \multirow[t]{2}{*}{$\begin{array}{l}\text { Organic } \\
\text { carbon } \\
\left(\mathrm{g} \mathrm{kg}^{-1}\right)\end{array}$} & \multirow[t]{2}{*}{$\begin{array}{l}\mathrm{CaCO}_{3} \\
\left(\mathrm{~g} \mathrm{~kg}^{-1}\right)\end{array}$} & \multirow{2}{*}{ 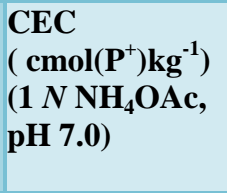 } & \multicolumn{4}{|c|}{ 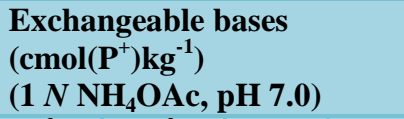 } & \multirow{2}{*}{$\begin{array}{l}\text { Base } \\
\text { saturation } \\
(\%) \\
\left(1 \mathrm{~N} \mathrm{NH}_{4}\right. \\
\text { OAc, pH 7.0) }\end{array}$} \\
\hline & & $\mathrm{H}_{2} \mathrm{O}$ & $\mathrm{I} N \mathrm{KCl}$ & & & & & & $\mathrm{Mg}^{2+}$ & $\mathrm{Na}^{+}$ & $\mathrm{K}^{+}$ & \\
\hline \multicolumn{13}{|c|}{ Pedon 1. Vertic Haplustepts (Plain) } \\
\hline Ap & $0.00-0.15$ & 8.1 & 7.7 & 0.93 & 0.43 & 5.5 & 51.2 & 39.7 & 8.7 & 1.7 & 0.4 & 99 \\
\hline 2Bw1 & $0.15-0.41$ & 8.0 & 7.9 & 0.78 & 0.34 & 14.5 & 44.6 & 33.5 & 8.2 & 0.7 & 0.4 & 96 \\
\hline 3Bw2 & $0.41-0.64$ & 8.3 & 8.0 & 0.78 & 0.22 & 15.5 & 43.2 & 30.7 & 9.6 & 1.2 & 0.3 & 97 \\
\hline 4Bw3 & $0.64-0.90$ & 8.5 & 8.2 & 0.77 & 0.18 & 18.5 & 42.1 & 28.5 & 10.9 & 1.9 & 0.2 & 99 \\
\hline 5Bw4 & $0.90-1.07$ & 8.6 & 8.2 & 0.74 & 0.16 & 2.5 & 40.9 & 24.0 & 14.7 & 1.7 & 0.2 & 99 \\
\hline Bw5 & $1.07-1.50+$ & 8.7 & 8.3 & 0.85 & 0.21 & 7.5 & 40.8 & 24.6 & 9.4 & 1.9 & 0.2 & 88 \\
\hline \multicolumn{13}{|c|}{ Pedon 2. Typic Ustorthents (Plain) } \\
\hline Ap & $0.00-0.23$ & 8.6 & 8.2 & 0.89 & 0.18 & 5.5 & 42.5 & 35.0 & 7.2 & 0.1 & 0.1 & 99 \\
\hline A1 & $0.23-0.52$ & 8.4 & 8.1 & 0.20 & 0.13 & 6.5 & 33.6 & 21.7 & 2.7 & 0.0 & 0.4 & 74 \\
\hline A2 & $0.52-0.72$ & 8.1 & 8.1 & 0.12 & 0.10 & 15.5 & 21.8 & 15.4 & 4.3 & 0.1 & 0.4 & 92 \\
\hline $\mathrm{Cr}$ & 0.72 & \multicolumn{11}{|c|}{ Weathered gneiss } \\
\hline \multicolumn{13}{|c|}{ Pedon 3. Vertic Haplustepts (Plain) } \\
\hline Ap & $0.00-0.18$ & 8.4 & 7.9 & 0.12 & 0.40 & 33.5 & 42.3 & 32.75 & 6.5 & 0.6 & 0.4 & 95 \\
\hline Bw1 & $0.18-0.43$ & 8.5 & 7.9 & 0.28 & 0.31 & 21.5 & 42.3 & 30.75 & 6.8 & 0.7 & 0.4 & 91 \\
\hline Bw2 & $0.43-0.67$ & 8.3 & 7.9 & 0.49 & 0.09 & 37.5 & 41.9 & 36.75 & 3.8 & 1.0 & 0.3 & 99 \\
\hline Bw3 & $0.67-0.91$ & 8.1 & 7.6 & 0.59 & 0.35 & 25.5 & 42.7 & 37.75 & 3.5 & 1.1 & 0.3 & 99 \\
\hline $\mathbf{C r}$ & 0.91 & \multicolumn{11}{|c|}{ Dolomite } \\
\hline \multicolumn{13}{|c|}{ Pedon 4. Typic Haplustepts (Upland) } \\
\hline Ap & $0.00-0.19$ & 8.2 & 7.3 & 0.29 & 0.75 & 6.5 & 42.6 & 35.0 & 5.6 & 0.0 & 0.6 & 97 \\
\hline Bw1 & $0.19-0.48$ & 8.0 & 7.2 & 0.66 & 0.66 & 7.5 & 41.7 & 32.5 & 3.0 & 0.1 & 0.2 & 86 \\
\hline 2Bw2 & $0.48-0.86$ & 7.9 & 7.7 & 0.57 & 0.21 & 7.5 & 38.4 & 24.5 & 4.4 & 0.2 & 0.1 & 76 \\
\hline 3Bw3 & $0.86-1.10$ & 8.5 & 7.8 & 0.35 & 0.12 & 8.5 & 40.4 & 22.8 & 2.1 & 0.1 & 0.2 & 63 \\
\hline 4Bw4 & $1.10-1.40$ & 8.2 & 7.8 & 0.25 & 0.27 & 8.5 & 50.1 & 28.7 & 2.2 & 0.1 & 0.2 & 62 \\
\hline $\mathrm{Cr}$ & 1.40 & \multicolumn{11}{|c|}{ Granite containing plagioclase feldspars with pegmatite veins } \\
\hline \multicolumn{13}{|c|}{ Pedon 5. Typic Haplustepts (Plain) } \\
\hline Ap & $0.00-0.21$ & 8.2 & 7.7 & 0.25 & 0.58 & 27.5 & 50.9 & 30.1 & 2.5 & 0.1 & 0.8 & 66 \\
\hline A1 & $0.21-0.61$ & 8.1 & 7.9 & 0.35 & 0.20 & 26.5 & 44.7 & 28.6 & 7.8 & 1.3 & 0.4 & 85 \\
\hline Bw1 & $0.61-1.01$ & 8.6 & 8.1 & 0.28 & 0.25 & 25.5 & 37.0 & 22.9 & 9.4 & 2.9 & 0.3 & 96 \\
\hline Bw2 & $1.01-1.40$ & 8.7 & 8.1 & 0.22 & 0.04 & 22.5 & 28.3 & 21.2 & 2.5 & 2.9 & 0.4 & 95 \\
\hline Bw3 & $1.40-2.00$ & 8.5 & 7.9 & 0.24 & 0.07 & 28.5 & 38.6 & 26.8 & 3.4 & 2.4 & 0.4 & 86 \\
\hline $\mathrm{Cr}$ & 2.00 & \multicolumn{11}{|c|}{ Granite containing plagioclase feldspars with pegmatite veins } \\
\hline \multicolumn{13}{|c|}{ Pedon 6. Lithic Ustorthents (Upland) } \\
\hline Ap & $0.00-0.18$ & 8.3 & 8.1 & 0.27 & 0.44 & 19.5 & 41.8 & 27.6 & 4.4 & 1.8 & 0.9 & 83 \\
\hline A1 & $0.18-0.39$ & 8.4 & 8.3 & 0.24 & 0.32 & 17.5 & 51.2 & 21.5 & 2.8 & 1.4 & 0.1 & 51 \\
\hline A2 & $0.39-0.50$ & 8.6 & 8.3 & 0.48 & 0.04 & 10.5 & 46.9 & 20.8 & 3.0 & 1.3 & 0.0 & 54 \\
\hline $\mathrm{Cr}$ & 0.50 & \multicolumn{11}{|c|}{ Granite containing plagioclase feldspars with pegmatite veins } \\
\hline Pedon 7. Ty & ic Ustorthen & s (Upla & ind) & & & & & & & & & \\
\hline Ap & $0.00-0.20$ & 8.2 & 8.0 & 0.12 & 0.29 & 17.5 & 30.2 & 27.6 & 4.44 & 1.8 & 0.9 & 83 \\
\hline A1 & $0.20-0.50$ & 8.0 & 7.9 & 0.13 & 0.14 & 16.5 & 28.6 & 21.5 & 2.82 & 1.4 & 0.1 & 51 \\
\hline A2 & $0.50-0.87$ & 8.1 & 8.0 & 0.14 & 0.16 & 16.5 & 25.1 & 20.8 & 3.0 & 1.3 & 0.0 & 54 \\
\hline A3 & $0.87-1.00$ & 8.1 & 8.1 & 0.14 & 0.13 & 14.5 & 26.2 & 20.4 & 2.70 & 0.6 & 0.3 & 91 \\
\hline $\mathrm{Cr}$ & 1.00 & Gran & & Ing pragi & Clase iefus & , pass witi & pegmatite veins & & & & & \\
\hline
\end{tabular}


Table.3 Physical characteristics of the soils

\begin{tabular}{|c|c|c|c|c|c|c|c|}
\hline $\begin{array}{l}\text { Pedon } \\
\text { No. \& } \\
\text { Horizon }\end{array}$ & $\begin{array}{l}\text { Depth } \\
\text { (m) }\end{array}$ & $\begin{array}{l}\text { Sand } \\
(\%) \\
(0.05- \\
2.0 \\
\text { mm) }\end{array}$ & $\begin{array}{l}\text { Silt } \\
(\%) \\
(0.002- \\
0.05 \\
\text { mm) }\end{array}$ & $\begin{array}{l}\text { Clay } \\
(\%) \\
(<0.002 \\
\text { mm) }\end{array}$ & $\begin{array}{l}\text { Bulk } \\
\text { density } \\
\left(\mathrm{Mg} \mathrm{m}^{-3}\right)\end{array}$ & $\begin{array}{l}\text { Particle } \\
\text { density } \\
\left(\mathrm{Mg} \mathrm{m}^{-3}\right)\end{array}$ & $\begin{array}{l}\text { Water } \\
\text { holding } \\
\text { capacity } \\
(\%)\end{array}$ \\
\hline \multicolumn{8}{|c|}{ Pedon 1. Vertic Haplustepts (Plain) } \\
\hline Ap & $0.00-0.15$ & 25.65 & 30.96 & 43.39 & 1.40 & 2.52 & 38 \\
\hline 2Bw1 & $0.15-0.41$ & 26.79 & 34.58 & 38.63 & 1.21 & 2.50 & 47 \\
\hline 3Bw2 & $0.41-0.64$ & 20.20 & 45.40 & 34.40 & 1.59 & 2.36 & 60 \\
\hline $4 \mathrm{Bw3}$ & $0.64-0.90$ & 28.57 & 38.17 & 33.26 & 1.57 & 2.14 & 48 \\
\hline 5Bw4 & $0.90-1.07$ & 28.74 & 40.86 & 25.40 & 1.36 & 2.60 & 70 \\
\hline Bw5 & $1.07-1.50+$ & 30.15 & 42.57 & 27.28 & 1.59 & 2.70 & 49 \\
\hline \multicolumn{8}{|c|}{ Pedon 2. Typic Ustorthents (Plain) } \\
\hline Ap & $0.00-0.23$ & 50.85 & 21.37 & 27.78 & 1.52 & 2.68 & 37 \\
\hline A1 & $0.23-0.52$ & 51.54 & 23.34 & 25.12 & 1.62 & 2.23 & 37 \\
\hline A2 & $0.52-0.72$ & 51.78 & 30.69 & 17.53 & 1.47 & 2.30 & 34 \\
\hline $\mathrm{Cr}$ & 0.72 & \multicolumn{6}{|c|}{ Weathered gneiss } \\
\hline \multicolumn{8}{|c|}{ Pedon 3. Vertic Haplustepts (Plain) } \\
\hline Ap & $0.00-0.18$ & 19.79 & 31.33 & 48.88 & 1.69 & 2.32 & 54 \\
\hline Bw1 & $0.18-0.43$ & 23.42 & 25.16 & 51.42 & 1.78 & 2.36 & 57 \\
\hline Bw2 & $0.43-0.67$ & 27.79 & 21.66 & 50.55 & 1.70 & 2.50 & 55 \\
\hline Bw3 & $0.67-0.91$ & 18.86 & 32.71 & 48.43 & 1.26 & 2.35 & 59 \\
\hline $\mathrm{Cr}$ & 0.91 & \multicolumn{6}{|c|}{ Dolomite } \\
\hline \multicolumn{8}{|c|}{ Pedon 4. Typic Haplustepts (Upland) } \\
\hline Ap & $0.00-0.19$ & 36.21 & 24.70 & 39.09 & 1.21 & 2.36 & 47 \\
\hline Bw1 & $0.19-0.48$ & 38.43 & 29.48 & 32.09 & 1.26 & 2.07 & 47 \\
\hline 2Bw2 & $0.48-0.86$ & 32.41 & 47.11 & 20.48 & 1.19 & 2.14 & 45 \\
\hline 3Bw3 & $0.86-1.10$ & 49.21 & 26.41 & 24.38 & 1.44 & 2.17 & 41 \\
\hline 4Bw4 & $1.10-1.40$ & 44.21 & 14.47 & 41.32 & 1.44 & 2.38 & 41 \\
\hline $\mathrm{Cr}$ & 1.40 & \multicolumn{6}{|c|}{ Granite containing plagioclase feldspars with pegmatite veins } \\
\hline \multicolumn{8}{|c|}{ Pedon 5. Typic Haplustepts (Plain) } \\
\hline Ap & $0.00-0.21$ & 16.62 & 46.78 & 36.60 & 1.32 & 2.50 & 36 \\
\hline A1 & $0.21-0.61$ & 45.65 & 23.91 & 30.44 & 1.61 & 2.20 & 41 \\
\hline Bw1 & $0.61-1.01$ & 21.69 & 50.59 & 27.72 & 1.15 & 2.06 & 38 \\
\hline Bw2 & $1.01-1.40$ & 28.13 & 48.70 & 23.17 & 1.51 & 2.60 & 30 \\
\hline Bw3 & $1.40-2.00$ & 46.63 & 32.03 & 21.34 & 1.38 & 2.18 & 32 \\
\hline $\mathrm{Cr}$ & 2.00 & \multicolumn{6}{|c|}{ Granite containing plagioclase feldspars with pegmatite veins } \\
\hline \multicolumn{8}{|c|}{ Pedon 6. Lithic Ustorthents (Upland) } \\
\hline Ap & $0.00-0.18$ & 20.97 & 46.10 & 32.93 & 1.29 & 2.57 & 49 \\
\hline A1 & $0.18-0.39$ & 17.91 & 44.37 & 37.72 & 1.28 & 2.45 & 36 \\
\hline A2 & $0.39-0.50$ & 19.96 & 46.34 & 33.70 & 1.59 & 2.43 & 48 \\
\hline $\mathrm{Cr}$ & 0.50 & \multicolumn{6}{|c|}{ Granite containing plagioclase feldspars with pegmatite veins } \\
\hline \multicolumn{8}{|c|}{ Pedon 7. Typic Ustorthents (Upland) } \\
\hline Ap & $0.00-0.20$ & 43.64 & 30.24 & 26.12 & 1.43 & 53 & 27 \\
\hline A1 & $0.20-0.50$ & 52.79 & 24.80 & 22.41 & 1.25 & 50 & 33 \\
\hline A2 & $0.50-0.87$ & 42.55 & 31.26 & 26.19 & 1.52 & 36 & 37 \\
\hline A3 & $0.87-1.00$ & 43.05 & 36.19 & 20.76 & 1.35 & 34 & 37 \\
\hline $\mathrm{Cr}$ & 1.00 & \multicolumn{6}{|c|}{ Granite containing plagioclase feldspars with pegmatite veins } \\
\hline
\end{tabular}


Table.4 Fertility status of the soils

\begin{tabular}{|c|c|c|c|c|c|c|c|c|c|}
\hline \multirow{3}{*}{$\begin{array}{l}\text { Pedon } \\
\text { No. \& } \\
\text { Horizon }\end{array}$} & \multirow{3}{*}{$\begin{array}{l}\text { Depth } \\
\text { (m) }\end{array}$} & \multicolumn{4}{|c|}{ Available macronutrients } & \multicolumn{4}{|c|}{ Available micronutrients } \\
\hline & & $\mathrm{N}$ & $\mathrm{P}$ & $\mathrm{K}$ & \multirow{2}{*}{$\begin{array}{l}\mathrm{S} \\
\mathrm{mg} \mathrm{kg}\end{array}$} & \multirow{2}{*}{\multicolumn{4}{|c|}{ 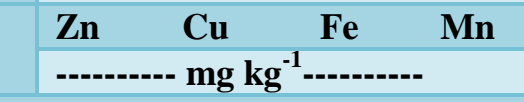 }} \\
\hline & & \multicolumn{3}{|c|}{-------- $\mathrm{kg} \mathrm{ha}^{-1}---------$} & & & & & \\
\hline \multicolumn{10}{|c|}{ Pedon 1. Vertic Haplustepts (Plain) } \\
\hline Ap & $0.00-0.15$ & 439 & 32 & 205 & 38 & 0.57 & 2.69 & 2.70 & 10.36 \\
\hline 2Bw1 & $0.15-0.41$ & 361 & 36 & 325 & 30 & 0.35 & 1.38 & 0.93 & 5.25 \\
\hline 3Bw2 & $0.41-0.64$ & 329 & 45 & 274 & 23 & 0.31 & 2.03 & 0.83 & 3.91 \\
\hline $4 B w 3$ & $0.64-0.90$ & 301 & 46 & 156 & 45 & 0.57 & 1.89 & 1.31 & 3.14 \\
\hline 5Bw4 & $0.90-1.07$ & 251 & 39 & 136 & 48 & 0.31 & 1.32 & 1.92 & 2.14 \\
\hline Bw5 & $1.07-1.50+$ & 221 & 40 & 169 & 25 & 0.23 & 1.69 & 0.91 & 3.92 \\
\hline \multicolumn{10}{|c|}{ Pedon 2. Typic Ustorthents (Plain) } \\
\hline Ap & $0.00-0.23$ & 321 & 33 & 122 & 18 & 0.31 & 0.77 & 0.58 & 3.94 \\
\hline A1 & $0.23-0.52$ & 285 & 66 & 152 & 23 & 0.25 & 0.46 & 0.56 & 3.47 \\
\hline A2 & $0.52-0.72$ & 263 & 73 & 162 & 21 & 0.20 & 0.22 & 0.42 & 2.70 \\
\hline $\mathrm{Cr}$ & 0.72 & \multicolumn{8}{|c|}{ Weathered gneiss } \\
\hline \multicolumn{10}{|c|}{ Pedon 3. Vertic Haplustepts (Plain) } \\
\hline Ap & $0.00-0.18$ & 390 & 36 & 389 & 24 & 0.30 & 1.11 & 1.11 & 2.36 \\
\hline Bw1 & $0.18-0.43$ & 351 & 39 & 337 & 26 & 0.40 & 1.19 & 0.81 & 5.12 \\
\hline Bw2 & $0.43-0.67$ & 317 & 40 & 289 & 38 & 0.44 & 1.32 & 0.56 & 4.25 \\
\hline Bw3 & $0.67-0.91$ & 293 & 54 & 230 & 18 & 0.50 & 0.99 & 0.62 & 2.98 \\
\hline $\mathrm{Cr}$ & 0.91 & \multicolumn{8}{|c|}{ Dolomite } \\
\hline \multicolumn{10}{|c|}{ Pedon 4. Typic Haplustepts (Upland) } \\
\hline Ap & $0.00-0.19$ & 373 & 46 & 278 & 28 & 0.36 & 1.81 & 0.67 & 6.13 \\
\hline Bw1 & $0.19-0.48$ & 343 & 78 & 216 & 20 & 0.31 & 1.29 & 0.49 & 5.88 \\
\hline 2Bw2 & $0.48-0.86$ & 309 & 57 & 106 & 21 & 0.28 & 1.42 & 0.56 & 2.76 \\
\hline 3Bw3 & $0.86-1.10$ & 287 & 71 & 197 & 20 & 0.22 & 1.75 & 0.73 & 3.44 \\
\hline 4Bw4 & $1.10-1.40$ & 254 & 41 & 207 & 28 & 0.31 & 0.85 & 0.43 & 6.16 \\
\hline $\mathrm{Cr}$ & 1.40 & \multicolumn{8}{|c|}{ Granite containing plagioclase feldspars with pegmatite veins } \\
\hline \multicolumn{10}{|c|}{ Pedon 5. Typic Haplustepts (Plain) } \\
\hline Ap & $0.00-0.21$ & 313 & 59 & 409 & 68 & 0.48 & 1.26 & 0.77 & 6.47 \\
\hline A1 & $0.21-0.61$ & 279 & 49 & 365 & 45 & 0.43 & 0.72 & 0.38 & 5.04 \\
\hline Bw1 & $0.61-1.01$ & 252 & 45 & 243 & 58 & 0.34 & 1.09 & 0.52 & 5.44 \\
\hline Bw2 & $1.01-1.40$ & 235 & 44 & 354 & 41 & 0.27 & 0.93 & 0.55 & 5.17 \\
\hline Bw3 & $1.40-2.00$ & 376 & 39 & 338 & 42 & 0.19 & 0.70 & 0.53 & 2.62 \\
\hline $\mathrm{Cr}$ & 2.00 & \multicolumn{8}{|c|}{ Granite containing plagioclase feldspars with pegmatite veins } \\
\hline \multicolumn{10}{|c|}{ Pedon 6. Lithic Ustorthents (Upland) } \\
\hline Ap & $0.00-0.18$ & 293 & 57 & 287 & 45 & 0.32 & 2.12 & 0.99 & 9.76 \\
\hline A1 & $0.18-0.39$ & 223 & 33 & 114 & 30 & 0.31 & 1.98 & 0.67 & 9.26 \\
\hline A2 & $0.39-0.50$ & 190 & 48 & 44 & 55 & 0.28 & 1.52 & 0.60 & 8.80 \\
\hline $\mathrm{Cr}$ & 0.50 & \multicolumn{8}{|c|}{ Granite containing plagioclase feldspars with pegmatite veins } \\
\hline \multicolumn{10}{|c|}{ Pedon 7. Typic Ustorthents (Upland) } \\
\hline Ap & $0.00-0.20$ & 310 & 38 & 310 & 22 & 0.36 & 0.59 & 0.59 & 6.90 \\
\hline A1 & $0.20-0.50$ & 279 & 36 & 260 & 19 & 0.23 & 0.50 & 0.43 & 3.87 \\
\hline A2 & $0.50-0.87$ & 271 & 17 & 252 & 20 & 0.16 & 0.68 & 0.63 & 4.35 \\
\hline A3 & $0.87-1.00$ & 221 & 13 & 239 & 13 & 0.20 & 0.32 & 0.39 & 2.48 \\
\hline $\mathrm{Cr}$ & 1.00 & \multicolumn{8}{|c|}{ Granite containing plagioclase feldspars with pegmatite veins } \\
\hline
\end{tabular}


Table.5 Soil productivity index and capability classes

\begin{tabular}{|c|c|c|c|c|c|}
\hline Soil site & Physiography & $\begin{array}{l}\text { Soil productivity } \\
\text { (P) }\end{array}$ & $\begin{array}{l}\text { Potential soil } \\
\text { productivity } \\
\text { (P') }\end{array}$ & $\begin{array}{c}\text { Coefficient of } \\
\text { Improvement } \\
\text { (P/P') }\end{array}$ & $\begin{array}{c}\text { Capability sub- } \\
\text { classes } \\
(\%)\end{array}$ \\
\hline P1 Pasupula & Plains & $\begin{array}{c}14.87 \\
\text { (Extremely Poor) }\end{array}$ & $\begin{array}{c}29.16 \\
\text { (Average) }\end{array}$ & 1.96 & IIs \\
\hline P2 Yerragudi Palem & Plains & $\begin{array}{c}26.44 \\
\text { (Average) }\end{array}$ & $\begin{array}{c}64.80 \\
\text { (Good) }\end{array}$ & 2.45 & IIIs \\
\hline P3 Palukuru & Plains & $\begin{array}{c}27.91 \\
\text { (Average) }\end{array}$ & $\begin{array}{c}54.72 \\
\text { (Good) }\end{array}$ & 1.96 & IIIsw \\
\hline P4 Mittepalle & Uplands & $\begin{array}{c}31.49 \\
\text { (Average) }\end{array}$ & $\begin{array}{c}58.32 \\
\text { (Good) }\end{array}$ & 1.85 & IIIes \\
\hline P5 Kapulapalle & Plains & $\begin{array}{c}33.46 \\
\text { (Average) }\end{array}$ & $\begin{array}{c}65.61 \\
\text { (Excellent) }\end{array}$ & 1.96 & IIIs \\
\hline P6 Illuru Kothapeta & Uplands & $\begin{array}{c}13.38 \\
\text { (Extremely Poor) }\end{array}$ & $\begin{array}{c}52.49 \\
\text { (Good) }\end{array}$ & 3.92 & IVes \\
\hline P7 Gulamnabipeta & Uplands & $\begin{array}{c}45.90 \\
\text { (Good) }\end{array}$ & $\begin{array}{c}90.00 \\
\text { (Excellent) }\end{array}$ & 1.96 & IVes \\
\hline
\end{tabular}




\section{Micronutrients}

The DTPA extractable $\mathrm{Zn}$ ranged from 0.16 to $0.57 \mathrm{mg} \mathrm{kg}^{-1}$ soil. Vertical distribution of $\mathrm{Zn}$ exhibited little variation with depth. Considering $0.6 \mathrm{mg} \mathrm{kg}^{-1}$ as critical level (Lindsay and Norvell 1978) these soils were deficient in available zinc. The low available zinc was possibly due to high soil $\mathrm{pH}$ values which might be resulted in the formation of insoluble compounds of zinc or insoluble calcium zincate (Jagdish Prasad et al., 2009). All the pedons were found to be sufficient in available copper ( 0.22 to $\left.2.69 \mathrm{mg} \mathrm{kg}^{-1}\right)$ as all the values were well above the critical limit of $0.20 \mathrm{mg} \mathrm{kg}^{-1}$ soil as suggested by Lindsay and Norvell (1978). The DTPA extractable Fe content varied from 0.38 to $2.70 \mathrm{mg} \mathrm{kg}^{-1}$ soil. According to critical limit of $4.5 \mathrm{mg} \mathrm{kg}^{-1}$ of Lindsay and Norvell (1978), the soils were deficient in available iron. The distribution of available iron in all the pedons did not show a definite pattern but this was abruptly decreased. It might be due to accumulation of organic carbon in the surface horizons. Surface horizons had higher concentration of DTPA-extractable Fe due to higher organic carbon. Available Manganese varied from 2.14 to $10.36 \mathrm{mg} \mathrm{kg}^{-1}$ soil (Table 4). According to critical limit of $1.0 \mathrm{mg} \mathrm{kg}^{-1}$ of Lindsay and Norvell (1978), the soils were sufficient in available manganese and almost decreased with depth which might be due to higher biological activity and organic carbon in the surface horizons, the higher content of available Mn in surface soils was attributed to the chelating of organic compounds released during the decomposition of organic matter left after harvesting of crop.

\section{Productivity and productive potentials}

The land associated with pedons 1 and 6 were classified under extremely poor. soil productivity of pedon 1 having the constraints of heavy texture and improper drainage and low organic carbon status changed to average and good, respectively by the application of farm yard manure, green manuring and inclusion of legumes in rotation, and texture can be improved by mixing with tank silt year after year. Pedon 6 associated with poor class having limitations of wetness (drainage) and physical soil characteristics like texture, soil depth and fertility characteristics like $\mathrm{pH}$ and organic carbon, changed to good by addition of tank silt, fertilizers, amendments ad inclusion of legumes in rotation (Table 1). Soil productivity was average for pedons 2,3 , 4 and 5 having the limitations of shallow depth, low soil moisture content and low organic matter content. On the use of good agro-techniques, land improvement practices such as deepening of top soil by ridging, deep ploughing or breaking up of soil pan, the productivity potentiality of pedons 2,3 and 4 could be brought to the level of good (Gangopadhyay et al., 2012). Pedon 7 was classified under good class of soil productivity. The productivity potentiality was noted as excellent for pedons 5 and 7 with the adequate use of soil and water conservation and soil fertility management practices. Quantitatively the coefficient of improvements in the present study ranged from 1.85 to 3.92. Based on the Riquier's parametric approach, the soils of Banaganapalle mandal of Kurnool district had three categories for cultivation of crops i.e. average, good and excellent (Table 5).

In conclusion, morphological, physical and physico-chemical properties of soils in semiarid Agro-ecological region of Banaganapalle mandal were slightly alkaline to strongly alkaline, non-saline, low to medium in organic carbon and CEC was low to medium and exchange complex was dominated by $\mathrm{Ca}^{2+}$. Soils were low to medium in available nitrogen, medium to high in available phosphorus, low to high in available potassium and high in available sulphur. The 
soils falls under IIs, IIIs, IIIsw, IIIes and IVes land capability sub-classes and suitable land use plan was also suggested for sustaining yields of the crops in Banaganapalle mandal. Evaluation of soils by actual and potential productivity indicated the present productivity of soils and potential productivity of soils after making improvements. Furthermore the coefficient of improvement $(\mathrm{Ci})$ indicating judicious use of organic manures in combination with inorganic fertilizers not only paves the way for achieving sustainable yields of crops but also maintains health of our finite soil without deterioration for future generations.

\section{References}

AIS\&LUS. 1970 Soil Survey Manual, All India Soil and Land Use Survey Organisation, IARI, New Delhi, pp. 163.

Ashokkumar, H.P. and Jagdish Prasad. 2010. Some typical sugarcane growing soils of Ahmadnagar district of Maharashtra. Their characterization, classification and nutritional status of soils and plants. Journal of the Indian Society of Soil Science 58, 257-266.

Gangopadhyay, S.K., Dipak Sarkar, Sahoo, A.K. and Singh, S.K. 2012. Soils of the rainfed region of West Bengal and their Productivity potential appraisal. Journal of the Indian Society of Soil Science 60 (2): 83-91.

Jagdish Prasad, Ray, S.K., Gajbhiye, K.S. and Singh, S.R. 2009. Soils of selsura research farm in wardha district, Maharastra and their suitability for crops. Agropedology 19, 84-91.

Klingebiel, A.A. and Montgomery, P.H. 1966. Agricultural Hand Book No. 210, USDA, Washington.

Leelavathi, G.P., Naidu, M.V.S., Ramavatharam, N and Karuna Sagar, G. 2010. Clay mineralogy of soils formed on granite-gneiss of Chittoor district, Andhra Pradesh Journal of the Indian Society of Soil Science 58(4): 376-383.

Lindsay, W.L. and Norvell, W.A. 1978. Development of DTPA soil test for zinc, iron, manganese and copper. Soil Science Society of America Journal 42, 421-428.

Nayak, D. C., Sarkar, D. and Das, K. 2002. Forms and distribution of pedogenic iron, aluminum and manganese in some Benchmark soils of West Bengal. Journal of the Indian Society of Soil Science 50, 89-93.

Patil, R.B. and Jagdish Prasad. 2004. Characteristics and classification of some Sal (Shorea robusta) supporting soils in Dindori district of Madhya Pradesh. Journal of the Indian Society of Soil Science 52, 119-124.

Raghuwanshi, S.R.S., Tiwari, S.C., Prabha, S., Raghuwanshi, O.P.S., Sasode, D.S. and Umat, R. (2011) Characterization of salt-affected soils of Bhind district of Madhya Pradesh. Journal of the Indian Society of Soil Science 59, 388-391.

Riquier, J., Bramo, D.L. and Cornet, J.P. 1970. A New System of Soil Appraisal in terms of Actual and Potential Productivity. FAO, Soil Resource Development and Conservation Service, Land and Water Development Division, FAO, Rome. pp. 88-99.

Sarkar, D (2011)Geo-informatics for appraisal and management of land resources towards optimizing agricultural production in the country-issues and strategies. Journal of the Indian Society of Soil Science 59 (Supplement), pp S35-S48.

Sarkar, D., Gangopadhyay, S.K. and Velayutham, M. 2001. Soil toposequence relationship and classification in lower outlier of Chhotanagpur plateau. Agropedology 11, 29-36. 
Satish Kumar, Y.S. and Naidu, M.V.S. 2012. Characteristics and classification of soils representing major landforms in Vadamalapeta mandal of Chittoor district, Andhra Pradesh. Journal of the Indian Society of Soil Science 60, 63-67.

Seghal, J.L., Mandal, D.K., Mandal, C. and Vadivelu, S. 1992. Agro-ecological Regions of India. NBSS Publ. No.24, NBSS\&LUP (ICAR), Nagpur.

Sharma, S.S., Totawat, K.L. and Shyampura, R.L. 2004 Characterization and classification of salt-affected soils of southern Rajasthan. Journal of the Indian Society of Soil Science 52, 209213.

Sharma, V.K. and Anil Kumar. 2003. Characterization and classification of the soil of upper Maul Khad catchment in wet temperate zone of Himachal Pradesh. Agropedology 13, 39-49.

Singh, I.S. and Agarwal, H.P. 2005. Characterization, genesis and classification of rice soils of Eastern Region of Varanasi, Uttar Pradesh. Agropedology 15, 29-38.

Soil Survey Division Staff. 2000. Soil Survey
Manual (Indian Print), USDA Handbook 18, US Govt. Printing Office, Washington.

Soil Survey Staff. 2010. Keys to Soil Taxonomy, $11^{\text {th }}$ edition, USDA, Natural Resource Conservation Service, Washington, DC.

Thangasamy, A., Naidu, M.V.S., Ramavatharam, N. and Raghava Reddy, C. 2005. Characterization, classification and evaluation of soil resources in Sivagiri micro - watershed of Chittoor district in Andhra Pradesh for sustainable land use planning. Journal of the Indian Society of Soil Science 53, 11-21.

Walia, C.S. and Rao, Y.S. 1997. Characteristics and classification of some soils of Trans-Yamuna plains. Journal of the Indian Society of Soil Science 45, 156-162.

Wani, S.P., Rockstrom, J. and Oweis, T. 2009. Rainfed agriculture - Past trends and future prospects. In Rainfed Agriculture: Unlocking the Potential, CABI, UK. Pp. 1-35.

\section{How to cite this article:}

Geetha Sireesha, P.V. and Naidu, M.V.S. 2020. Production Potential Appraisal: A Case Study in Banaganapalle Mandal of Kurnool District in Andhra Pradesh, India. Int.J.Curr.Microbiol.App.Sci. 9(06): 787-798. doi: https://doi.org/10.20546/ijcmas.2020.906.101 